\title{
Enhancement of Raman light scattering in dye-labeled cell membrane on metal-containing conducting polymer film
}

\author{
H. V. Grushevskaya*, N. G. Krylova and I. V. Lipnevich \\ Physics Department, Belarusan State University, \\ 4 Nezalezhnasti Ave., 220030 Minsk, Belarus \\ *grushevskaja@bsu.by \\ T. I. Orekhovskaja, V. P. Egorova and B. G. Shulitski \\ Belarusan State University of Informatics and Radioelectronica, \\ 6 P. Brovki Str., 220013 Minsk, Belarus \\ Accepted 29 February 2016 \\ Published 31 March 2016
}

\begin{abstract}
An enhanced Raman spectroscopy method based on a plasmon resonance in ultrathin metal-containing LB-film deposited on nanoporous anodic alumina supports has been proposed. This material has been utilized to enhance Raman scattering of light in fluorescent-labeled subcellular membrane structures. It has been shown that the plasmon resonance between vibrational modes of the organometallic complexes monolayers and dye-labeled subcellular structures happens. It makes possible to detect interactions between living cell monolayers and an extracellular matrix.
\end{abstract}

Keywords: Raman spectroscopy method; ultrathin metal-containing LB-film; monolayers; cytoplasmic membrane; conducting multi-walled carbon nanotubes.

PACS numbers: 87.64.Je, 68.47.Pr, 81.07.Pr, 87.16.Dg

\section{Introduction}

At present, it has been found out that an interaction of biological cells with any extracellular-matrix occurs through subcellular structures formed by integrins and called focal adhesions. ${ }^{1,2}$ The binding of focal adhesions with surface of substrate can be affected by globular glycoproteins, for example, a plasma lactoferrin. ${ }^{3}$ Lactoferrins bind preferably to double-stranded (ds) than to single-stranded DNAs. Because of this, one can assume that the ds-DNA is a regulatory molecule in the process of cell attachment/detachment during active adhesion of focal contacts. This is possible if the cytoplasmic membrane $(\mathrm{cm})$ DNA exists. ${ }^{4}$ The presence of nucleic acids in the plasma membrane is provided by both an intercellular transport by gap junction channels ${ }^{5,6}$ and microtubules ${ }^{7}$ and an extracellular transport of 
apoptotic DNA fragments. ${ }^{8}$ But, it has been shown that the plasma membrane contains small molecules of a specific cmDNA, sequence of which is identical to telomere DNA-sequence. ${ }^{9}$ To date, there are no experimental facts concerning the role of the specific cmDNA. A presence of this cmDNA has to affect the process of active adhesion. However, the detection of such interaction is hampered due to the nanosize of the focal adhesions with height about $10-15 \mathrm{~nm}^{1}$ and due to the dependence of DNA Raman spectra on the DNA protonation. ${ }^{10} \mathrm{~A}$ sensitive method of nanosizestructure visualization is the surface-enhanced Raman spectroscopy (SERS). Owing to the closeness of metallic nanoparticles, oppositely charged parts of particle surfaces produce electric dipoles, quadrupoles and so on, which oscillate in resonance, and a phenomenon of giant Raman scattering is observed. ${ }^{11}$ Due to the high intensity of this giant resonance, the intensities of Raman spectrum bands depend on random distribution of nanoparticles and, because of this, change from one experiment to another in a random manner. The variability of SERS spectrum intensity is a serious disadvantage of SERS-method based on metallic nanoparticles, because it demands to create an ideal layer of periodically arranged nanoparticles.

In this paper, we use high-ordered metal-containing LB-films of organometallic complexes from a conducting oligomer of thiophene-pyrrole series and conducting multi-walled carbon nanotubes (MWCNT) to enhance the Raman scattering. We will demonstrate that these graphene-like materials can screen the electric field of electrically charged double layer on the interface.

The goal of the paper is to establish both a screening effect of conducting metal-containing LB-film deposited on nanoporous anodic alumina (AOA) and a phenomenon of plasmon resonance in the oscillating electric field of screened electrically charged double layer on the interface water/condensed state, and to develop a SERS-method based on these effects and to apply it in Raman spectroscopy of adhered living cell monolayers.

\section{Materials and Methods}

\subsection{Reagents}

Salt $\mathrm{Fe}\left(\mathrm{NO}_{3}\right)_{3} \cdot 9 \mathrm{H}_{2} \mathrm{O}$ (Sigma-Aldric, USA), hydrochloric acid, deionized water were used to prepare subphases. Iron-containing films were fabricated from amphiphilic oligomer of thiophene derivatives with chemically bounded hydrophobic 16-link hydrocarbon chain: 3-hexadecyl-2,5-di(thiophen-2-yl)-1H-pyrrole (H-DTP, H-dithionilepyrrole). ${ }^{12}$ All salt solutions have been prepared with deionized water with resistivity of $18.2 \mathrm{M} \Omega \cdot \mathrm{cm}$.

MWCNTs with diameters ranging from 2.0 to $5 \mathrm{~nm}$ and length of $\sim 5-10 \mu \mathrm{m}$ were obtained by the method of chemical vapor deposition (CVD-method). ${ }^{13}$ MWCNTs were covalently modified by carboxyl groups and non-covalently functionalized by stearic acid molecules.

RNA and protein fractions in high-purity ds-DNA $\left(1.03 \mathrm{mg} / \mathrm{ml}\right.$ in $10^{-5} \mathrm{M}$ $\mathrm{Na}_{2} \mathrm{CO}_{3}$ buffer medium (TE-buffer)) were less than $0.1 \%$ (optical density ratio 
$\mathrm{D}_{160} / \mathrm{D}_{230}=2.378$ and $\mathrm{D}_{160} / \mathrm{D}_{280}=1.866$, respectively). This DNA was utilized to fabricate hydrophobic (reverse) micelles of stearic acid with ds-DNA inside them by ultrasonic treatment of a blend from stearic acid with ds-DNA in hexane.

C6 rat glioma cells obtained from culture collection of Institute of Epidemiology and Microbiology (Minsk, Belarus) were grown on Fe-containing LB-DTPfilms with or without aligned MWCNT arrays in a Dulbecco's modified Eagle medium (DMEM) (Sigma, USA) supplemented with 10\% fetal bovine serum and $1 \times 10^{-4} \mathrm{~g} / \mathrm{ml}$ gentamycin at $37^{\circ} \mathrm{C}$ in a humidified $5 \% \mathrm{CO}_{2}$ atmosphere. A cellular monolayer has been stained with $1 \mu \mathrm{M}$ propidium iodide (PI, Sigma, USA) for five minutes before spectral studies were carried out.

Membranes of porous AOA have been used as a support for LB-films. Fabrication of AOA films membranes (thickness of $20.0 \mu \mathrm{m}$ ) was carried out by means of two-stage anodic treatment in $10 \%$ sulfuric acid solution at voltage of $10 \mathrm{~V}$ and temperature of $2^{\circ} \mathrm{C}$. The diameter of $\mathrm{AOA}$ pore was equal to $10 \mathrm{~nm}$.

All used materials belong to class of analytical pure reagents.

\subsection{Methods}

A Langmuir-Blodgett (LB) monolayer formation was carried out on an automated hand-made Langmuir trough with controlled deposition on a substrate, and with computer user interface working under Microsoft Windows operational system. Control of the surface tension was performed by a highly sensitive resonant inductive sensor. The Y-type transposition of monolayers on supports was performed by their vertical dipping. The complexes Fe(II)DTP $\mathrm{DT}_{3}$ of high-spin Fe(II) with thiophene-pyrrole ligands were synthesized by LB-technique at compression of H-dithionilepyrrole molecules on the surface of subphase with salts of three valence Fe. ${ }^{12}$ Horizontally and vertically arranged LB-MWCNT-bundles can be fabricated from the multi-walled CNTs. ${ }^{14}$ We use the Langmuir-Blodgett technique to fabricate new biocompatible layered nanoheterostructures consisting of two MWCNT LB-monolayers and/or 5-, 7-monolayer LB-film of the organometallic complexes.

Spectral studies in visible range were carried out using a confocal micro-Raman spectrometer Nanofinder HE ("LOTIS-TII", Tokyo, Japan-Belarus) by laser excitation at wavelength $532 \mathrm{~nm}$ and vertical spatial resolution $150 \mathrm{~nm}$.

\section{Screening Depending on Charge State of Mixed-Valence Complexes}

Coordination complexes of Fe are shown in Fig. 1(a) and 1(b). Metal atoms in the LB-monolayer of the iron complexes $\mathrm{FeDTP}_{3}$ in charge state " $3+$ " are placed in the monolayer plane. Since high-spin iron complexes are dome-shaped, the LBmonolayer of the iron complexes in charge state " $2+$ " represents itself as a graphenelike material decorated by a monolayer of adhesive iron atoms (adatoms), as shown in Fig. 1(c). 
(a)

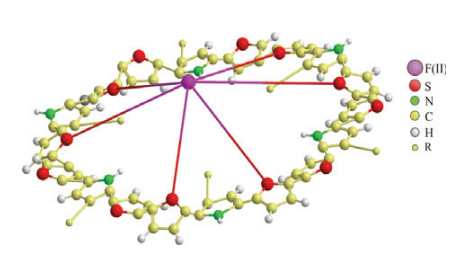

(b)

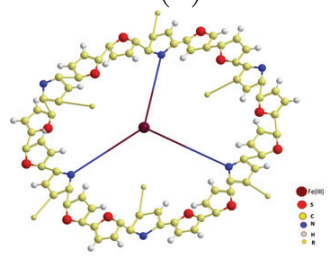

(c)

Fig. 1. Structure formulas of complexes (a) $\mathrm{Fe}(\mathrm{II}) \mathrm{DTP}_{3}$ and (b) $\mathrm{Fe}(\mathrm{III}) \mathrm{DTP}_{3}$. (c) A scheme of LB-monolayer.

Let us consider the screening in true two-dimensional (2D) semimetals. As known, ${ }^{15-17}$ free charge carriers at the Fermi level in these materials are absent. In single-pole approximation for small momenta $p$ the condition of emerging of plasmon resonance can be found from the following dependency of dielectric permeability $\epsilon(p, \omega)$ on a frequency $\omega$ :

$$
\epsilon(p, \omega) \approx 1-\frac{1}{\left(\omega^{2} / \omega_{\text {plasmon }}^{2}\right)-p^{2} r_{\mathrm{D}}^{2}}, \quad p \ll 1,
$$

where $r_{\mathrm{D}}=\left(\sum_{a}\left(e_{a}^{2} n_{a} / \epsilon_{0} k_{\mathrm{B}} T\right)\right)^{-1 / 2}$ is the Debye screening radius, $\omega_{\text {plasmon }}=$ $\left(\sum_{a}\left(e_{a}^{2} n_{a} / \epsilon_{0} m_{a}\right)\right)^{1 / 2}$ is a plasmon frequency, $a$ is a type of the charge carriers, $n_{a}, m_{a}$ and $e_{a}$ are a density, a mass and a charge of $a$-th type, respectively; $\epsilon_{0}$ is the dielectric constant, $T$ is the temperature, $k_{\mathrm{B}}$ is the Boltzmann constant. Pseudofermion charge carriers in bipolar graphene-like two-dimensional material are massless: $m_{c}=0$ and their charge density is vanishing: $n_{c} \rightarrow 0$ in Dirac point $K\left(K^{\prime}\right)$ of a Brillouin zone. Due to the fact that $r_{\mathrm{D}} \rightarrow \infty$ and plasmon frequency gets a finite value $\omega_{\text {plasmon }}^{2}=2\left(e^{2} / \epsilon_{0}\right)$, the right side of the condition (1) is always positive and, respectively, plasmon resonance in $2 \mathrm{D}$ system is absent.

Now, one can examine decorated 2D semimetals. In Coulomb field of adatoms, a band structure of the monolayer acquires the energy gap $E_{g}$ and on Fermi level there appears non-zero charge-carrier density $n_{c}=\delta n_{\mathrm{F}}<1$. Therefore, the dielectric permeability $\epsilon_{\mathrm{LB}}(p, \omega)$ of the decorated system is determined by the following expression

$$
\epsilon_{\mathrm{LB}}(p, \omega) \approx 1-\frac{1}{\left(\epsilon_{0} \omega^{2} E_{g} /\left(2 e^{2} \delta n_{\mathrm{F}}\right)\right)-2 p^{2} \frac{2 e^{2} \delta n_{\mathrm{F}}}{\epsilon_{0} k_{B} T}}, \quad p \ll 1 .
$$

The expression (2) can acquire zero and negative values, corresponding to plasmon resonance. Figures 2(a) and 2(b) demonstrate the absence of screening and, respectively, plasmon oscillations for non-decorated 2D Dirac metals.

An enhancement of Raman light scattering in the organometallic LB-film, on whose surface the MWCNT-containing monolayers have been deposited, is shown in Fig. 3(a). This is possible, if MWCNTs are decorated by the monolayer of high-spin Fe-adatoms, that makes MWCNTs to be able to screen an electromagnetic field. 
(a)

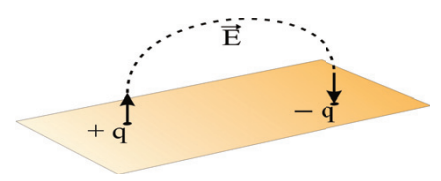

(b)

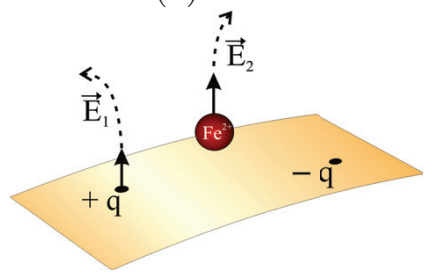

Fig. 2. Origin of screening effect in 2D Dirac metal (a) after decorating (b).

(a)

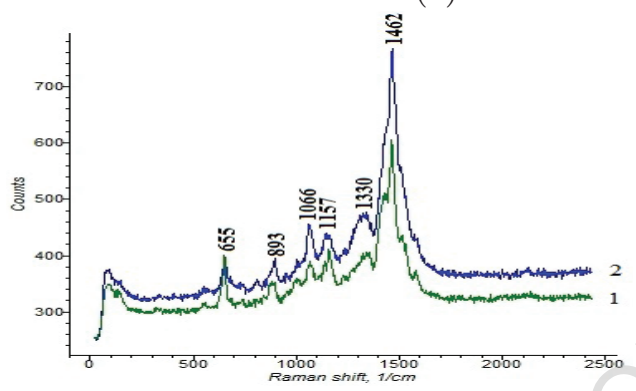

(b)

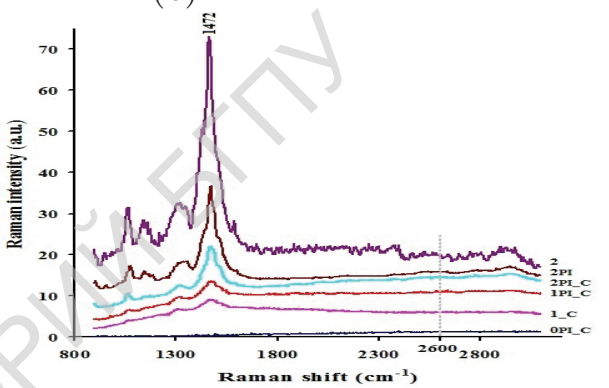

Fig. 3. (a) Raman spectra of LB-films from five monolayers of $\mathrm{FeDTP}_{3}$ (1) coated by two monolayers of MWCNTs with stearic acid (2) in air. Laser excitation at $\lambda=532 \mathrm{~nm}$ with power $1.2 \mathrm{~mW}$. (b) Raman spectra of intensity integrated over whole sample in water medium for cells on glass (curve 0PI_C) and on five monolayers Fe-containing LB-DTP-films (curves 1_C, 1PI_C) coated by two monolayers of MWCNTs with stearic acid (curves 2, 2PI, 2PI_C). "PI" denotes the presence of $1 \mu \mathrm{M}$ propidium iodide in the cuvette, "C" denotes the presence of cellular monolayer on the LB-film. Laser excitation at $\lambda=532 \mathrm{~nm}$ with power $0.06 \mathrm{~mW}$.

These experimental results prove that one of Fe charge state in $\mathrm{FeDTP}_{3}$ is a state "+2". A Raman spectrum of $\mathrm{FeDTP}_{3}$ in Table 1 has oscillations 1342, 1348, 1410 and $1432 \mathrm{~cm}^{-1}$, which are absent in the original H-dithionilepyrrole spectrum. These oscillations arise from Fe atom periodically leaving the ligand monolayer plane and coming back when changing the valence state of Fe atom from coordinate state II to III and vice versa. The assumption, that these oscillations are due to the mixedvalence state of the metal center is confirmed by the presence of more high-frequency oscillation $1432 \mathrm{~cm}^{-1}$ of thiophene ring in the Raman spectrum. Because of that, we call this oscillation as the oscillation of metal-center valence. Protonation of the LBfilms, placed in water, leads to shift of Raman bands into high-frequency region, for example, for 2-subst pyrrole Quadrant stretch from 1462 to $1472 \mathrm{~cm}^{-1}$, according Table 1. A laser plasmon mode for the LB-film is represented by plasmon oscillations in the frequency regions $1650-2800 \mathrm{~cm}^{-1}$ and $1575-2300 \mathrm{~cm}^{-1}$ for samples placed in air and water, respectively, in Fig. 3. The frequencies of charge-density oscillations for free charge carriers in the LB-film are shown in Table 2. 
Table 1. Characteristic molecular vibrations, observed in the Raman spectrum of 5-, 7-monolayer LB film.

\begin{tabular}{|c|c|c|}
\hline Band center, $\mathrm{cm}^{-1}$ & $\begin{array}{l}\text { Region for vibration } \\
\text { of similar molecular } \\
\text { group, } \mathrm{cm}^{-1}\end{array}$ & Assignment \\
\hline 556 & 525 & ring in-plane deformation ${ }^{18}$ \\
\hline 653 & 586 & ring in-plane deformation ${ }^{18}$ \\
\hline 686 & 686 & thiophene ring in-plane deformation ${ }^{19}$ p. 64 \\
\hline 741 & $750-690$ & C-S in thiophene, ${ }^{19}$ p. 64 \\
\hline 804 & $808,990-700$ & thiophene ring in-plane deformation ${ }^{18,20}$ p. 58 \\
\hline Shoulder at 844 & 844 & thiophene $\operatorname{ring}^{21}$ \\
\hline 893 & $893,990-700$ & $\begin{array}{l}\text { trans hexadecyl-chain vibration, } \\
\text { thiophene } \text { ring }^{22} \text { p. } 118,{ }^{20} \text { p. } 58\end{array}$ \\
\hline 1080,1138 & 1063,1138 & $\begin{array}{l}\text { hexadecyl-chain trans } \mathrm{C}-\mathrm{C}-\mathrm{C} \\
\text { out-of-phase stretches }^{22} \text { p. } 154,140\end{array}$ \\
\hline 1158 & 1148 & $\mathrm{Cl}-\mathrm{O}$ vibration, ${ }^{1922}$ p. 170 \\
\hline 1231 & 1236 & $\mathrm{CH}$ bending ${ }^{18}$ \\
\hline 1310 & 1310 & inter-ring $\mathrm{C}-\mathrm{C}$ vibration ${ }^{23}$ \\
\hline 1342 & 1357 & semi-circle stretch of thiophene ring $^{22}$ p. 98 \\
\hline 1348 & $1361-1345$ & 2-subst thiophene Semi-circle stretch ${ }^{22}$ p. 109 \\
\hline 1410 & $1420-1400$ & 2-subst pyrrole Semi-circle stretch ${ }^{22}$ p. 98 \\
\hline 1432 & $1455-1430$ & 2-subst thiophene Quadrant stretch ${ }^{22}$ p. 98 \\
\hline $1462^{*}, 1472^{* *}$ & $1475-1460$ & 2-subst pyrrole Quadrant stretch ${ }^{22}$ p. 98 \\
\hline 1515 & $1514-1540$ & 2-subst thiophene Quadrant stretch ${ }^{22}$ p. 98 \\
\hline 1586 & $1585-1607$ & $\mathrm{C}=\mathrm{C}$ stretching in pyrrole rings ${ }^{23}$ \\
\hline 2940 & $2940-2915$ & $\mathrm{CH}_{2}$ in alkanes ${ }^{21}$ p. 4 \\
\hline
\end{tabular}

*, ** — measurements of samples have been performed in air and water, respectively.

Table 2. Frequency regions for oscillations of free-charge-carriers density in metal-containing dithionilepyrrole LB-films.

\begin{tabular}{ll}
\hline Region or Band center, $\mathrm{cm}^{-1}$ & \multicolumn{1}{c}{ Assignment } \\
\hline $1575-2300$ (water), 1650-2800 (air) & $\begin{array}{l}\text { plasmon oscillations } \\
\text { oscillations of metal-center valence }\end{array}$ \\
\hline
\end{tabular}

Thus, an enhancement of Raman light scattering by the Fe-containing organometallic LB-film is stipulated by both the plasmon and metal-center-valence oscillations. Further, we will demonstrate results in Raman light scattering enhancement in sites of biological-cell adhesion to support.

\section{Screening Effects of Organometallic LB-Films}

\subsection{Metal-containing LB-film enhancement of Raman light scattering in hydrated complexes}

An electrically charged double layer arises on an interphase boundary which includes the ultrathin LB-film. This layer is similar to a plane capacitor. Since a distance between plates of such capacitor is very small, its electric field strength is large. A high value of low-frequency dielectric permeability of water is due to the water ionization in this double layer with following formation of hydrated complexes of 
(a)

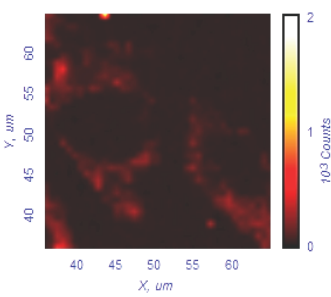

(b)

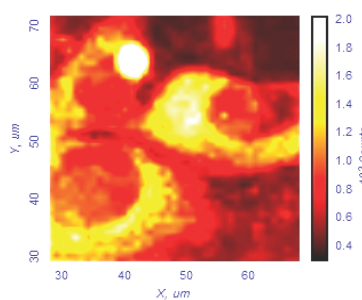

(c)

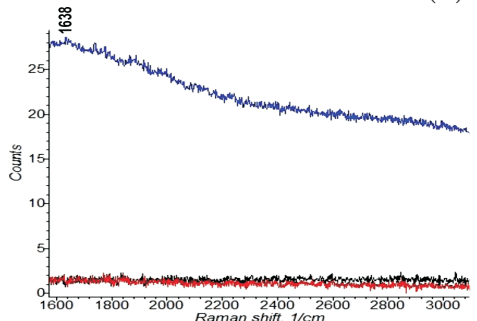

Fig. 4. (Color online) Typical confocal Raman images of living cells on glass (a) and on organometallic LB-film at presence of propidium iodide (b); registration at frequency $1600 \mathrm{~cm}^{-1}$. Raman spectra of intensity integrated over whole sample for cells on glass with (black) and without (red) PI and on LB-film with PI (blue) are shown in figure (c). The excitation laser wavelength was $532 \mathrm{~nm}$.

ions $\mathrm{OH}^{-}, \mathrm{H}^{+}$and impurity ions. The existence of such complexes impedes the ion recombination. A resonance of the free-charge density oscillations in the LBfilm at the eigenfrequencies of the hydration water oscillations enhances a process of hydrated complexes decay and, respectively, released ions recombine to neutral molecules $\mathrm{H}_{2} \mathrm{O}$ that results in decreasing of dielectric permeability of the medium.

Fluorescence of hydrated PI molecules is quenched by hydration water molecules. The resonance of oscillations of free-charge-carrier density in the metalcontaining LB-films at eigenfrequencies of hydrated shells leads to decay of complexes of PI with water and, respectively, to suppression of PI fluorescence quenching, as shown by comparison of curves in Fig. 3(b) and comparison of luminescence intensity of images of PI stained cells, which were grown on a glass and the conducting organometallic LB-film deposited on AOA in Fig. 4. Thus, LB-film screens the electric field of electrically charged double layer on the interphase boundary.

\subsection{Metal-containing LB-film enhancement of Raman light scattering in living cell monolayers}

Let us consider the screening effects of electrically charged double layer on the interface living-cell monolayer physiological medium. The ds-DNA inside the stearic acid micelles is similar to cmDNA. Therefore, to find a Raman spectrum of cmDNA for the cell under investigation, we carried out a comparison of characteristic frequencies of the Raman spectra for the living cell monolayers with a Raman spectrum of the ds-DNA inside stearic acid micelles in Fig. 5. According to Table 3, the spectrum of model micellar ds-DNA shifts relative to the Raman spectrum for DNA being at condition of $92 \%$ humidity, because, as it is known, ${ }^{10}$ the protonation of DNA causes a shift of DNA characteristic frequencies into high-frequency region. However, this shift is negligible compared to the shift of the corresponding bands of a Raman spectrum for water solutions of the ds$\mathrm{DNA}^{24}$ due to deprotonation of screened double layer at the condition of plasmon 
(a)

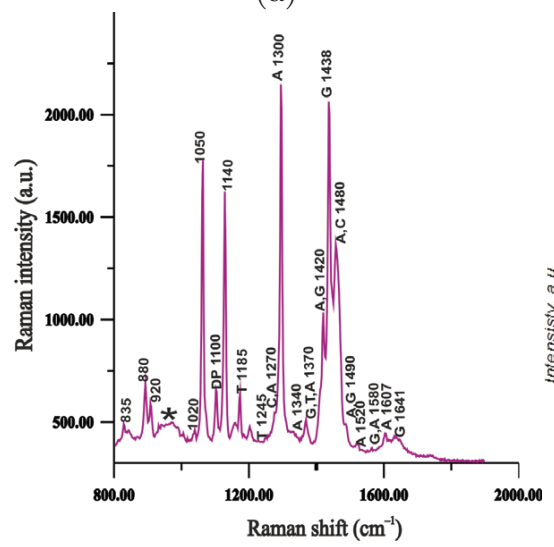

(b)

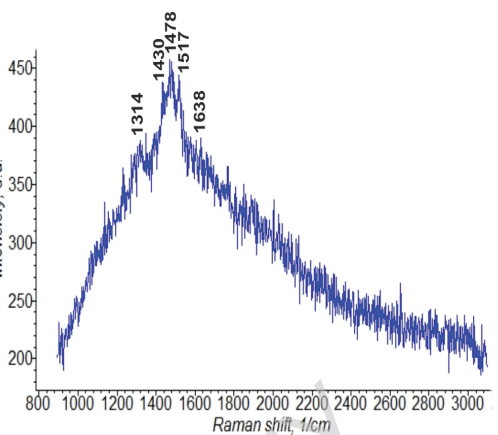

Fig. 5. (a) A Raman spectrum of micelles formed in hexane solution of mixture from stearic acid with ds-DNA and dripped on Si. The numbers indicate the characteristic frequencies of DNA vibrations; adenine, guanine, thymine and cytosine are designated by A, G, T and C, respectively; DP denotes phosphodiester bond $\langle\langle *\rangle\rangle$ indicates the laser peak of surface plasmons in Si. Laser power was $20 \mathrm{~mW}$. (b) A Raman spectrum of focal adhesion for living cell in monolayer stained with PI. Laser power was $0.06 \mathrm{~mW}$, excitation wavelength $532 \mathrm{~nm}$.

Table 3. Characteristic frequencies of Raman spectrum of light scattered in living cell monolayers and enhanced by metal-containing dithionilepyrrole LB-films.

\begin{tabular}{ll}
\hline $\begin{array}{l}\text { Band center, } \\
\mathrm{cm}^{-1}\end{array}$ & $\begin{array}{l}\text { Region for vibration of similar molecular } \\
\text { group, } \mathrm{cm}^{-1} \text { and assignment }\end{array}$ \\
\hline $1600-1638$ & $\begin{array}{l}\mathrm{N}=\mathrm{C} \text { stretching in G: } 1639,1641 \text { and in A: 1605, 1607 for DNA } \\
\text { at } 92 \% \text { humidity }\end{array}$ \\
& $\mathrm{NH}_{2}$ stretching in G: 1418,1438 for DNA \\
1478 & at $92 \%$ humidity ${ }^{10}$ and inside stearic acid micelles, respectively \\
\hline
\end{tabular}

resonance. According to Table 3, the characteristic frequencies of the Raman spectra of the cellular monolayer and the ds-DNA inside the micelle are close to each other. Moreover, the addition of PI into medium enhances the luminescence of the cellular monolayer. As known, ${ }^{25}$ besides PI molecules intercalation into double helix of DNA, PI molecules can complexify with DNA being situated in DNA minor groove. Fluorescence of hydrated complexes DNA/PI in minor groove is quenched by hydrate water molecules. Plasmon resonance at eigenfrequencies of hydrate shells leads to these complexes decay and, respectively, to suppression of fluorescence quenching of DNA/PI in water. Thus, the observed enhancement of fluorescence for the cell monolayer stained with PI is the luminescence of such DNA/PI complexes in Fig. 4.

Moreover, by virtue of the effective adhesion of DNA on a hydrophobic surface of graphene and graphene-like materials due to stacking interaction of their $\pi$-electrons, ${ }^{26-29}$ a coincidence of characteristic frequencies of the cell monolayer 
with DNA frequencies is also explained by the hydrophobicity of LB-film surface repulsing hydrophilic proteins, such as bovine serum albumin, that results in the small intensity of characteristic bands Amide-I (carbonyl group $\mathrm{C}=\mathrm{O}, 1650$ $1690 \mathrm{~cm}^{-1}$ ) and Amide-II (group N=C, $1450 \mathrm{~cm}^{-1}$ ) ${ }^{30}$ in the Raman spectrum. The fact, that we observe the enhanced Raman scattering of light in the cell cmDNA, is also proved by the absence of band Amide-I with maximum $1665 \mathrm{~cm}^{-1}$ for DNA, ${ }^{31}$ and the band $\left(\mathrm{C}=\mathrm{O}, 1615-1635 \mathrm{~cm}^{-1}\right)$ of linker histone $\mathrm{H} 1^{30}$ in their Raman spectrum.

\section{Conclusion}

So, a SERS in the living cell monolayer grown on ultrathin organometallic LBfilms is observed, in preference, on the characteristic frequencies of DNA. This is due to the facts that LB-film surface is hydrophobic and repulses the hydrophilic proteins, and cmDNA adheres effectively on the hydrophobic surfaces of graphene and graphene-like materials owing to the stacking interaction of their $\pi$-electrons.

\section{References}

1. V. Petit and J.-P. Thiery, Biol. Cell 92, 477 (2000).

2. A. W. Peterson et al., BMC Cell Biol. 10, 16 (2009).

3. K. R. Alugupalli et al., Oral Microbiol. Immunol. 9(3), 174 (1994).

4. R. M. Bennett et al., J. Clin. Invest. 71, 611 (1983).

5. S. Kurtenbach, S. Kurtenbach and G. Zoidl, Frontiers Physiol. 5, 82 (2014).

6. Z. Moinfar, H. Dambach and P. M. Faustmann, Frontiers Physiol. 5, 186 (2014).

7. Q. Wang et al., Theranostics 3(6), 395 (2013).

8. G. Servais et al., Ann. Rheum. Dis. 57, 606 (1998).

9. J. Cheng et al., Proc. Natl. Acad. Sci. USA 109, 10827 (2012).

10. G. P. Gigina and E. F. Oleinik, Uspechi Khimii 41, 475 (1972).

11. H. Ko, S. Singamaneni and V. V. Tsukruk, Small 4(10), 1576 (2008).

12. H. V. Grushevskaya, I. V. Lipnevich and T. I. Orekhovskaya, J. Mod. Phys. 4, 7 (2013).

13. V. Labunov et al., Quant. Electron. Optoelectron. 13, 137 (2010).

14. A. S. Egorov et al., J. Nonlin. Phen. Complex Syst. 15, 121 (2012).

15. S. V. Morozov, K. S. Novoselov and A. K. Geim, Phys. - Uspekhi 178, 776 (2008).

16. H. V. Grushevskaya and G. Krylov, J. Nonlin. Phen. Complex Syst. 17, 86 (2014).

17. H. V. Grushevskaya and G. G. Krylov, in Nanotechnology in the Security Systems, NATO Science for Peace and Security Series C: Environmental Security, ed. J. Bonča and S. Kruchinin (Springer Science+Business Media, Dordrecht, 2015), p. 21.

18. C. Constantino, J. Duff and R. Aroca, Spectrochimica Acta Part A 57, 1249 (2001).

19. K. Nakanishi, Infrared Spectra and Organic Compounds Structure (Mir, Moscow, 1965).

20. D. W. Brown, A. J. Floyd and M. Sainsbury, Spectroscopy of Organic Subctances (Mir, Moscow, 1992).

21. B. N. Tarasevich, IR-Spectra of Basis Classes of Organic Compounds (MSU, Moscow, 2012).

22. P. Larkin, Infrared and Raman Spectroscopy: Principles and Spectral Interpretation (Elsevier, Oxford, 2011). 
23. M. J. L. Santos, A. G. Brolo and E. M. Girotto, Electrochimica Acta 52, 6141 (2007).

24. A. V. Prieszhev, V. V. Tuchin and L. P. Shubochkin, Laser Diagnostics in Biology and Medcine (Nauka, Moscow, 1989).

25. P. O. Vardevanyan, R. A. Karapetyan and M. A. Parsadanyan, Biol. J. Armenia 4(62), 6 (2010).

26. A. S. Egorov et al., Herald Found. Basic Res. 3(14), 63 (2014).

27. V. Ermakov et al., Int. J. Mod. Phys. B 11, 827 (2007).

28. S. Kruchinin and T. Pruschke, Phys. Lett. A 378, 157 (2014).

29. V. E. Rodionov et al., Mater. Sci. 31, 232 (2013).

30. A. M. Poljanichko et al., Cytology 66(4), 316 (2014).

31. H. Nawaz et al., Analyst 135, 3070 (2010). 